\title{
Review of: "Upregulated PD-1 Signaling is an Important Antagonist to Glomerular Health in Aged Kidneys"
}

\author{
Liqiang Meng ${ }^{1}$ \\ 1 Beijing Medical University
}

Potential competing interests: The author(s) declared that no potential competing interests exist.

It is very interesting to explore the pathophysiological mechanism(s) of kidney aging, because we can make complete tissue regeneration possible via comprehensively understanding the mechanisms of aging. Reversing or delaying renal failure can make hundreds of millions of patients with chronic kidney disease get rid of disease problems. The authors have done a lot of works on the mechanisms of PD-1 in the kidney aging. It is a creative study to find the benefit of anti-PD-1 treatment to the aged kidney. I think it deserves to be recommended to the researchers. And I hope to know whether PD-1 affects the epigenetic changes of various inherent cells in the kidney. 